\title{
The vasorelaxant hormone relaxin induces changes in liver sinusoid microcirculation: a morphologic study in the rat
}

\author{
D Bani, S Nistri, S Quattrone, M Bigazzi ${ }^{1}$ and T Bani Sacchi \\ Department of Anatomy, Histology and Forensic Medicine, Section of Histology, University of Florence, V. le G. Pieraccini 6, I-50139 Florence, Italy \\ ${ }^{1}$ Prosperius Institute, V. le F.lli Rosselli 60-62, I-50123 Florence, Italy \\ (Requests for offprints should be addressed to D Bani, Dipartimento di Anatomia, Istologia e Medicina Legale, Sezione di Istologia, V. le G. Pieraccini 6 , \\ I-50139 Firenze, Italy; Email: bani@unifi.it)
}

\begin{abstract}
This study shows that specialized contractile endothelial cells exist in rat liver sinusoids which may be involved in the local control of hemodynamics and which are sensitive to vasoactive agents, including the vasorelaxant hormone relaxin. Male rats were treated with $10 \mu \mathrm{g}$ relaxin for 4 days; phosphate-buffered saline (PBS)-treated rats were the controls. For comparison, rats treated with relaxin together with the NO-synthase inhibitor $\mathrm{N}^{\omega}$-nitro-Larginine methyl ester (L-NAME), and rats treated with the vasodilator taurodeoxycholic acid or the vasoconstrictor ethanol were investigated. Liver fragments were studied morphologically and morphometrically. In the control rats,
\end{abstract}

peculiar contractile cells were present in the endothelial lining. These cells had abundant myofilaments and formed cytoplasmic blebs projecting into and often occluding the lumen. In the ethanol-treated rats, sinusoids were constricted and filled with cytoplasmic blebs. In the relaxintreated rats, sinusoids were markedly dilated and the cytoplasmic blebs nearly disappeared. Similar findings were observed in the taurodeoxycholic acid-treated rats. The effects of relaxin were blunted by L-NAME, suggesting that the relaxin action involves an $\mathrm{NO}$-mediated mechanism.

Journal of Endocrinology (2001) 171, 541-549

\section{Introduction}

The liver has two afferent blood supplies. One is the hepatic artery, which has primarily a nutritional function; the other is the portal vein, which collects the blood from the gastrointestinal tract and the spleen and is designed to allow exposure of absorbed substances and hormones to liver parenchyma where metabolic conversion of the nutrients takes place. The hepatic arterial and portal blood merge in a common pool into the sinusoids, which empty in the terminal hepatic venules. The hemodynamics of the hepatic microcirculation is thought to depend mainly on the activity of smooth muscle cells located in the walls of arterioles, terminal arterioles and 'precapillary sphincters'. The latter structures extend around the initial portion of the arterial capillaries which penetrate the thin layer of hepatocytes surrounding the portal space (the so-called limiting plate) and empty into the periportal sinusoids. These smooth muscle cells are responsive to nervous stimulation, hormones, metabolites, bile salts and vasoactive agents, and hence are regarded as the prime movers and regulators of pressure and flow in the microcirculatory units (Rappaport \& Schneiderman 1976). However, liver sinusoids can also greatly increase their caliber when needed, depending on intrinsic contractile mechanisms which had remained poorly understood for a long time
(Rappaport 1980). Earlier transillumination studies of the rat liver in vivo allowed postulation of the existence of large cells capable of contractile activity, located in an endothelial position in the arterial capillaries and at the entry and exit of the sinusoids, which might contribute to fine adjustments of the sinusoidal flow (McCuskey 1966, McCuskey et al. 1983). However, the technique used in those studies did not allow the authors to clarify the exact nature of these cells, thus leading more recent investigators of liver microcirculation to believe that the above in vivo observations require further substantiation (Campra \& Reynolds 1988). In the last decade, refinements of the liver transillumination technique (Bauer et al. 1995) have allowed the recognition of hepatic stellate cells, identified with fat-storing cells or Ito cells (Ramadori 1990), as possible physiologic regulators of sinusoidal blood flow, being able to constrict or dilate in response to vasoactive agents such as endothelins and carbon monoxide (Suematsu et al. 1995, Zhang et al. 1995, Bauer et al. 2000).

In the current study, we report on the characteristics of specialized contractile endothelial cells in the liver sinusoids of rats, likely involved in the regulation of blood microcirculation. These cells are sensitive to substances known to decrease or increase sinusoidal blood flow, such as ethanol (Hijioka et al. 1991) and bile acids 
(Pak \& Lee 1993) respectively, as well as to the vasorelaxant hormone relaxin (RLX). RLX is a peptide hormone typical of pregnancy (reviewed by Bani 1997) which has been shown to cause striking vasodilation in several organs (Vasilenko et al. 1986, Bani et al. 1988, 1995, Bigazzi et al. 1988, Bani Sacchi et al. 1995, Masini et al. 1997, Danielson et al. 1999). Among these organs, there are the mesenteric microvessels (Bigazzi et al. 1986), which are thought to play an important role in the regulation of portal blood flow and hence, indirectly, in liver perfusion (Rappaport 1973). In some target organs, such as the heart and the kidney, RLX exerts its vasodilatory action through the activation of endogenous nitric oxide (NO) biosynthesis (Bani Sacchi et al. 1995, Masini et al. 1997, Danielson et al. 1999). Of note, NO has been found to play a role in the regulation of intrahepatic portal circulation (Mittal et al. 1994). In the current study, we also show that RLX exerts a vasorelaxant action on liver sinusoids and that this action ostensibly involves an NO-mediated mechanism.

\section{Materials and Methods}

Nineteen male adult albino rats of the CD strain (Charles River, Como, Italy) weighing 300-350 g were used. In previous studies, RLX has been shown to have powerful vasodilatory action in both sexes (Bigazzi et al. 1986, 1988, Vasilenko et al. 1986, Bani et al. 1988, 1995, Bani Sacchi et al. 1995, Masini et al. 1997, Danielson et al. 1999). Therefore, in the current study, we used male rats to exclude any possible influence of endogenous RLX and ovarian steroids on the experimental results, as may have occurred if using cycling female rats. The rats were fed standard laboratory chow and water, available ad libitum, and were housed under a $12 \mathrm{~h}$ light: $12 \mathrm{~h}$ darkness photoperiod. The experimental protocol was designed in compliance with the Principles of Laboratory Animal Care (NIH publication No. 86-23, revised 1985) and the recommendations of the European Economic Community (86/609/ CEE), under the supervision of a competent local committee for the care and use of laboratory animals. The rats were acclimatized for 1 week before entering the experiments. They were then randomly distributed into five experimental groups and treated as follows.

Group $1(n=5)$ : rats treated for 4 days with two daily s.c. injections (at 0900 and $1600 \mathrm{~h}$ ), each containing $5 \mu \mathrm{g}$ purified porcine RLX in $0.5 \mathrm{ml}$ phosphate-buffered saline (PBS). The RLX used, purified according to the method of Sherwood \& O'Byrne (1974) and containing 2500$3000 \mathrm{U} / \mathrm{mg}$, was a generous gift of Dr O D Sherwood. Food ingestion has been found to increase blood flow to the gut and resultant hepatic blood flow (Shoemaker et al. 1963, Orrego et al. 1965). Therefore, to exclude any interference by digestive processes, the rats were fasted for 15-18 h after the last RLX injection. They were then anesthetized with pentobarbital (40 $\mathrm{mg} / \mathrm{kg}$ body weight) and killed by exsanguination.

Group $2(n=5)$ : rats treated with PBS alone, by the same route and administration schedule as for RLX injections, were used as controls. They were fasted, anesthetized and killed as for the RLX-treated rats.

Group $3(n=3)$ : rats treated with the NO synthase inhibitor $\mathrm{N}^{\omega}$-nitro-L-arginine methyl ester (L-NAME; Sigma, St Louis, MO, USA) before being injected with RLX as for group 1. The L-NAME solution $(0.5 \mathrm{~g} / 1$ in $\mathrm{H}_{2} \mathrm{O}$ ), freshly prepared every day, was given in the place of drinking water for 5 days, starting from the day before the first RLX injection. Intake of the L-NAME solution was approximately $200 \mathrm{ml} /$ day per animal. The rats were fasted, anesthetized and killed as those of group 1.

Group $4(n=3)$ : rats treated with $2 \mathrm{ml}$ ethanol $40 \%$ in water, given as a bolus by a cannula inserted into the stomach. Ten minutes later, upon the appearance of clear-cut behavioral signs of alcoholic inebriation, the rats were anesthetized with pentobarbital and killed by exsanguination.

Group $5(n=3)$ : rats anesthetized with pentobarbital and treated with taurodeoxycholic acid (TDC; $300 \mathrm{mg} / \mathrm{kg}$ body weight; Sigma), dissolved in $0.3 \mathrm{ml}$ physiologic saline and injected in the penile vein by slow infusion. Ten minutes after TDC injection, the rats were killed by exsanguination.

Daily consumption of food and water of the rats from groups 1 to 3 was measured. This was similar in all the groups, thus allowing us to exclude the possibility that differences of the hepatic sinusoid caliber between the three groups of rats might be dependent on different alimentary regimens.

At laparotomy, fragments of liver tissue were resected from the quadrate lobe. Some of them were fixed in $2.5 \%$ cold glutaraldehyde in $0 \cdot 1 \mathrm{M}$ cacodylate buffer, $\mathrm{pH} 7 \cdot 4$, postfixed in $1 \%$ osmium tetroxide in $0.1 \mathrm{M}$ phosphate buffer, $\mathrm{pH} 7 \cdot 4$, at room temperature, dehydrated in ethanol, passed through propylene-oxide and embedded in Epon 812. Semithin sections, $2 \mu \mathrm{m}$ thick, stained with toluidine blue-sodium tetraborate were used for light microscopic and morphometrical studies. Ultrathin sections stained with uranyl-acetate and lead citrate were examined under a JEM 1010 electron microscope (Jeol, Tokyo, Japan) at $80 \mathrm{kV}$. Other tissue fragments were placed in cryostat embedding medium (Pabisch, Milano, Italy) and quickly frozen in liquid nitrogen. Sections, $8 \mu \mathrm{m}$ thick, were cut from each specimen in a cryostat and used for detection of non-muscular actin in the endothelial cells. The sections were fixed in 3\% phosphate-buffered formaldehyde for $30 \mathrm{~min}$, washed thoroughly and treated with $0 \cdot 2 \%$ Triton X-100 (BDH, Poole, Dorset, UK) in PBS for $5 \mathrm{~min}$. After careful rinsing in PBS, the sections were incubated for $1 \mathrm{~h}$ with tetramethylrhodamineisothiocyanate (TRITC)-conjugated phalloidin (Sigma) dissolved in PBS to a final concentration of $0.4 \mu \mathrm{g} / \mathrm{ml}$. 

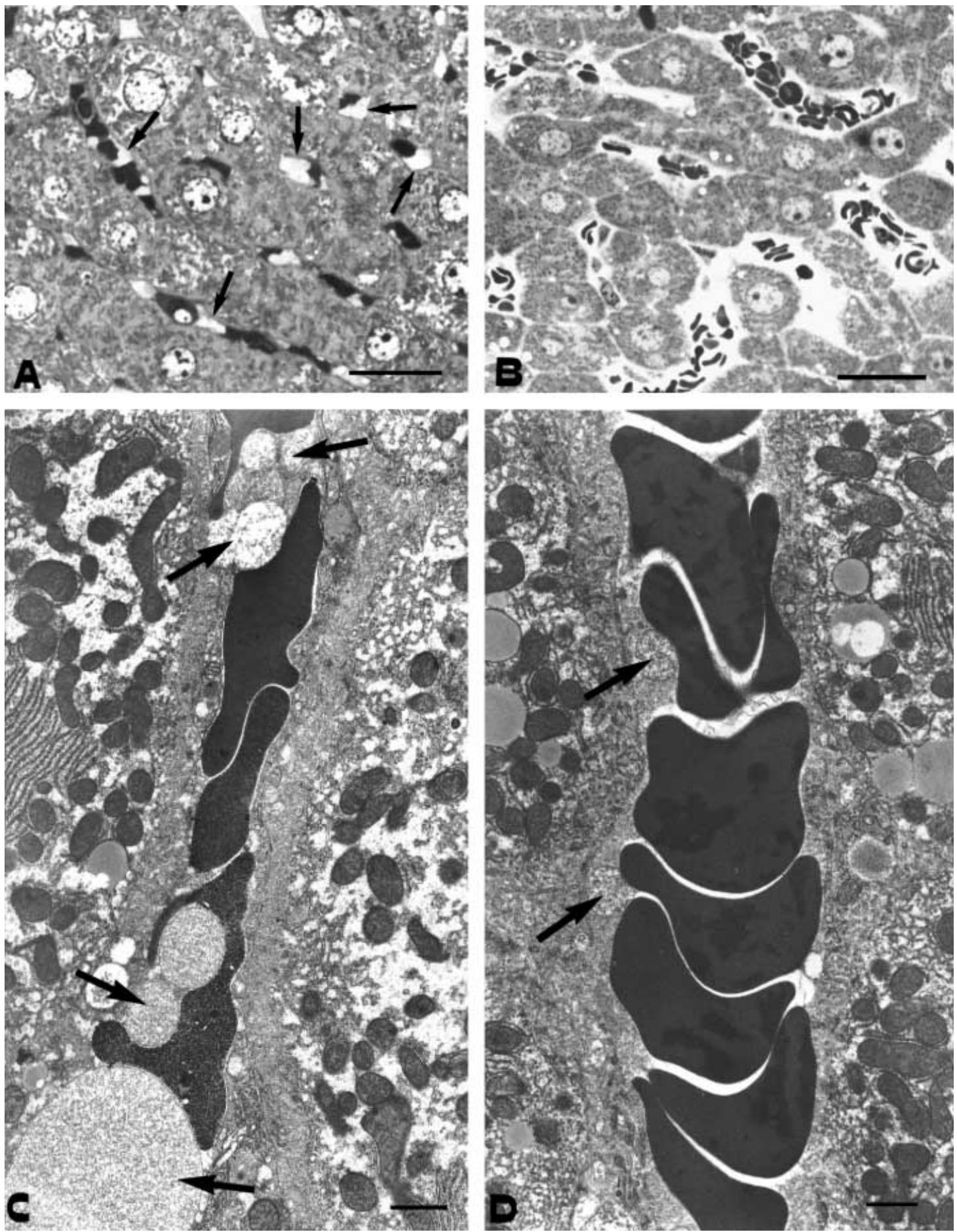

Figure 1 Light and electron microscopic features of liver sinusoids in the control rats (A and C) and the RLX-treated rats ( $B$ and $D)$. (A) Control rats: sinusoids are narrow and show numerous pale blebs (arrows) abutting into the lumen and sometimes occluding it. (B) RLX-treated rats: sinusoids are dilated and blebs are virtually absent. Semithin sections stained with toluidine blue-sodium tetraborate, $\times 515$, bars $=20 \mu \mathrm{m}$. (C) Control rats: the blebs in the sinusoids (arrows) contain a network of actin-like microfilaments. (D) RLX-treated rats: the sinusoidal endothelium is flattened and the blebs (arrows) are rare and small in size. Electron micrographs, $\times 8600$, bars $=1 \mu \mathrm{m}$. 

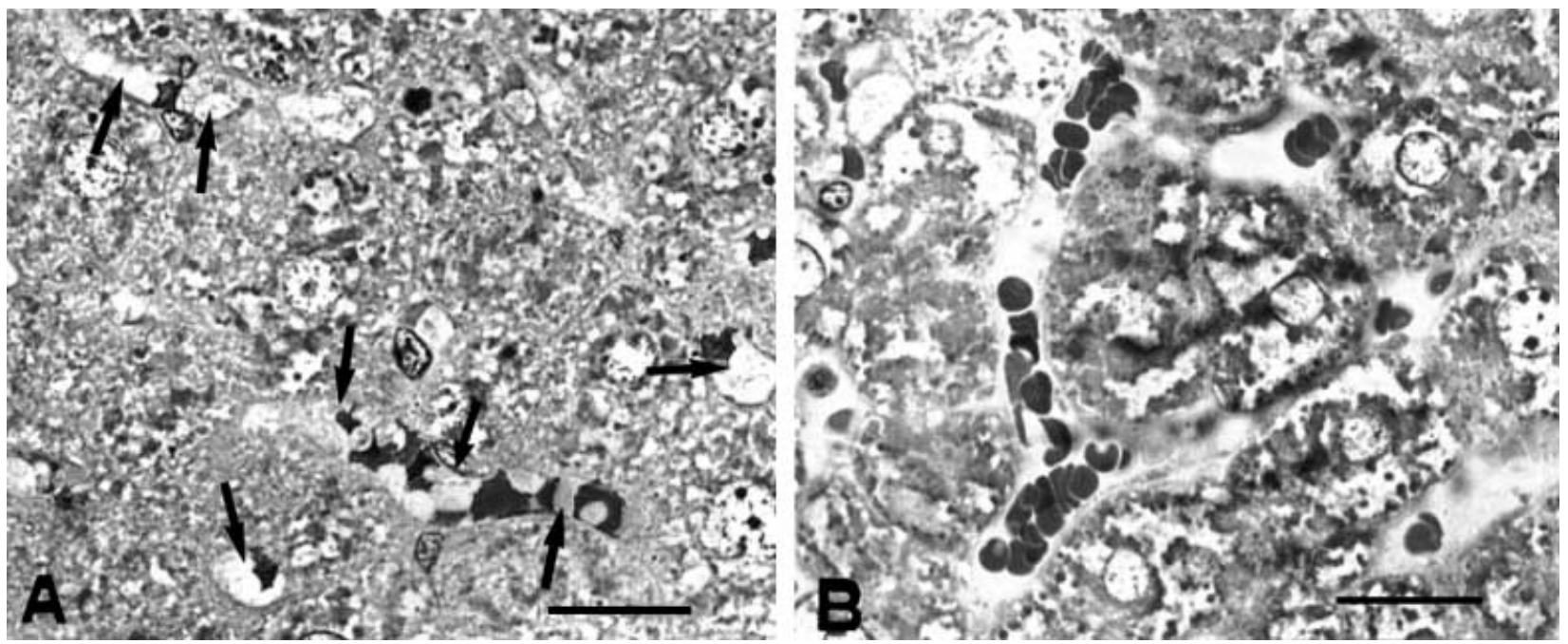

Figure 2 Light microscopic features of liver sinusoids in the ethanol-treated rats and the TDC-treated rats. (A) Ethanol-treated rats: most sinusoids are very constricted and their lumina occluded by blebs (arrows). (B) TDC-treated rats: sinusoids are dilated and blebs are virtually absent. Semithin sections stained with toluidine blue-sodium tetraborate, $\times 580$, bars $=20 \mu \mathrm{m}$.

Phalloidin is known to bind avidly and irreversibly to every actin isoform, including non-muscular actin. Negative controls were carried out on some sections by substituting the phalloidin solution with PBS. Sections were mounted in Gel/Mount aqueous medium (Biomeda, Foster City, CA, USA), and then observed and photographed under a Zeiss Axiophot UV-light microscope.

Computer-assisted morphometry of sinusoids was performed on the semithin sections as described previously for similar purposes (Norrby et al. 1996). Two different liver fragments per rat were used. For each fragment, five different microscopic fields were examined. The microscopic fields were photographed and glossy prints at a final magnification of $\times 1100$ were obtained. The sinusoidal profiles were accurately traced onto superimposed acetate sheets and then registered through a CCTV television camera (Sony, Tokyo, Japan) interfaced with an Apple Macintosh LC III personal computer through a Videospigot card (Supermac, Sunnyvale, CA, USA). The program used (1.49 Image Analysis Program; NIH, Bethesda, MD, USA) enables the area encircled by each profile to be measured. Values are expressed as mean area \pm S.E.M. Calculations were carried out using a GraphPad Prism 2.0 statistical program (GraphPad Software, San Diego, CA, USA). Distribution of values within groups was assessed to be log-normal. To create a Gaussian distribution, the values were transformed into their logarithms. Statistical comparison of differences between the different experimental groups was then made using one-way ANOVA test followed by Student-NewmanKeuls multiple comparison test. $P<0 \cdot 05$ was considered significant.

\section{Results}

Light microscopic examination of semithin sections stained with toluidine blue showed that the liver sinusoids of the control rats had pale bleb-like structures abutting into the lumen. Sometimes these blebs converged together to reduce or even to occlude the vascular lumen (Fig. 1A). By electron microscopy, the blebs showed varying dimensions and contained a more or less dense network of thin filaments resembling actin microfilaments (Fig. 1C). Conversely, in the RLX-treated rats, the liver sinusoids viewed under the light microscope appeared markedly dilated and the blebs were no more apparent or inconspicuous (Fig. 1B). By electron microscopy, the sinusoidal endothelium was mostly flattened, the blebs were an occasional finding and, when present, were very small (Fig. 1D). In the RLX-treated rats which received the NO-synthase inhibitor L-NAME, the liver sinusoids showed moderate dilation and the blebs appeared sparse (data not shown). In the rats treated with ethanol, many sinusoids were very constricted and filled with blebs occluding large tracts of their lumina (Fig. 2A). In the rats treated with TDC, the sinusoids were markedly dilated and very few or no intralumenal blebs could be observed (Fig. 2B).

Ultrastructural examination of the liver sinusoids of the control rats showed that the blebs corresponded to cytoplasmic protrusions of cells located in the endothelial lining. These cells were intermingled with typical endothelial cells, characteristically flattened and provided with fenestrations and pinocytotic vesicles, and showed peculiar features, i.e. a roundish cell body containing an extended smooth endoplasmic reticulum and several mitochondria 


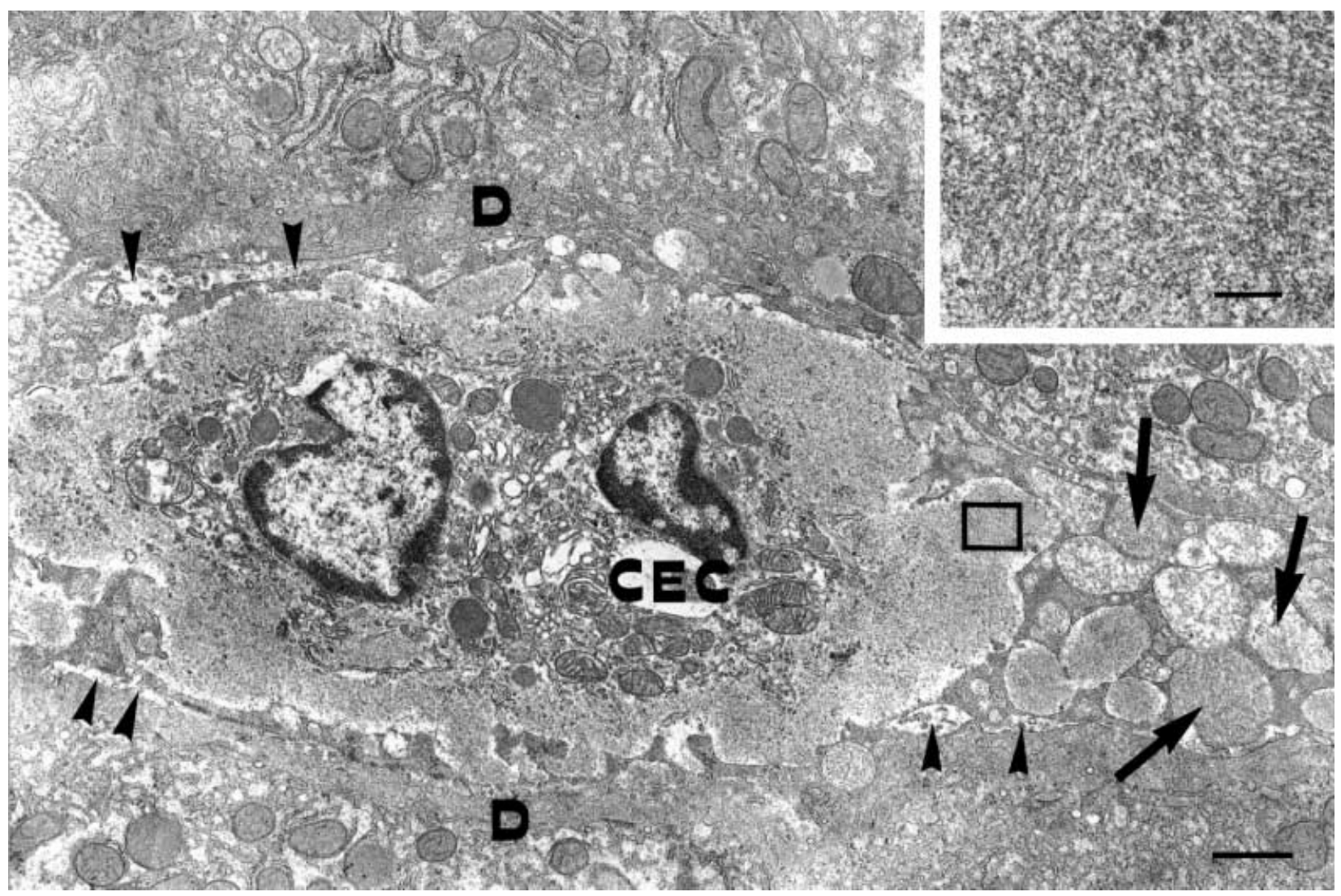

Figure 3 Representative electron micrograph of a CEC in a sinusoid of a control rat. The cell shows an extended smooth endoplasmic reticulum and several mitochondria concentrated in the paranuclear cytoplasm, and large bundles of actin-like microfilaments located peripherally (see detail in the inset). Numerous blebs, filled with microfilaments and devoid of organelles similar to the cell's peripheral cytoplasm, can be seen protruding in the sinusoidal lumen (arrows). The CEC is close to the common fenestrated endothelium of the sinusoid (indicated by arrowheads). D: Disse spaces. Electron micrograph, $\times 11700$, bar $=1 \mu \mathrm{m}$; inset, $\times 77700, b a r=100 \mathrm{~nm}$.

concentrated in the perinuclear cytoplasm, and large bundles of thin actin-like filaments located peripherally. Their cytoplasm expanded into long, irregular cytoplasmic protrusions, filled with filaments and devoid of organelles, in the form of blebs projecting toward the sinusoidal lumen (Fig. 3). Because of the unique features of these cells, we called them 'contractile endothelial cells' (CECs). The cytoplasmic processes of the CECs often adhered to the lumenal face of the typical endothelial cells and to their fenestrations, thus coming into direct contact with the Disse space. In the sinusoids located towards the portal spaces, CECs were numerous and their cytoplasmic blebs substantially reduced or even virtually occluded the sinusoidal lumen. In the sinusoids located towards the centrilobular vein, CECs and their blebs were sparse. CECs were also present at the inlet and outlet of the sinusoids, at the junction with the precapillary sphincters and the centrilobular vein respectively. CECs could be easily distinguished from hepatic stellate cells (Fig. 4), the latter being located in the Disse spaces and characterized by numerous lipid droplets, a feature that was never found in the CECs, and by unapparent contractile apparatus. CECs also differed from Kupffer cells (Fig. 4), which also prevailed in the sinusoids of the portal side and usually had the features of macrophages, i.e. an electron-dense cytoplasmic matrix, a well-developed rough endoplasmic reticulum, a large Golgi apparatus and varying amounts of lysosomes of heterogeneous size and inner structure. At variance with the sinusoids, the microvessels lying in the portal spaces and those penetrating the limiting plate, usually referred to as precapillary sphincters (Rappaport 1973), showed non-fenestrated endothelium with continuous basement membrane and were devoid of CECs. Typical smooth muscle cells (Fig. 4) were commonly found around these vessels, often forming myoendothelial junctions with the ablumenal aspect of the endothelial lining.

Additional insight into the peculiar features of CECs was provided by the studies with TRITC-labeled phalloidin, which showed that the endothelial blebs observed in the liver sinusoids of the control rats actually contained large amounts of actin (Fig. 5A). Of note, in the 

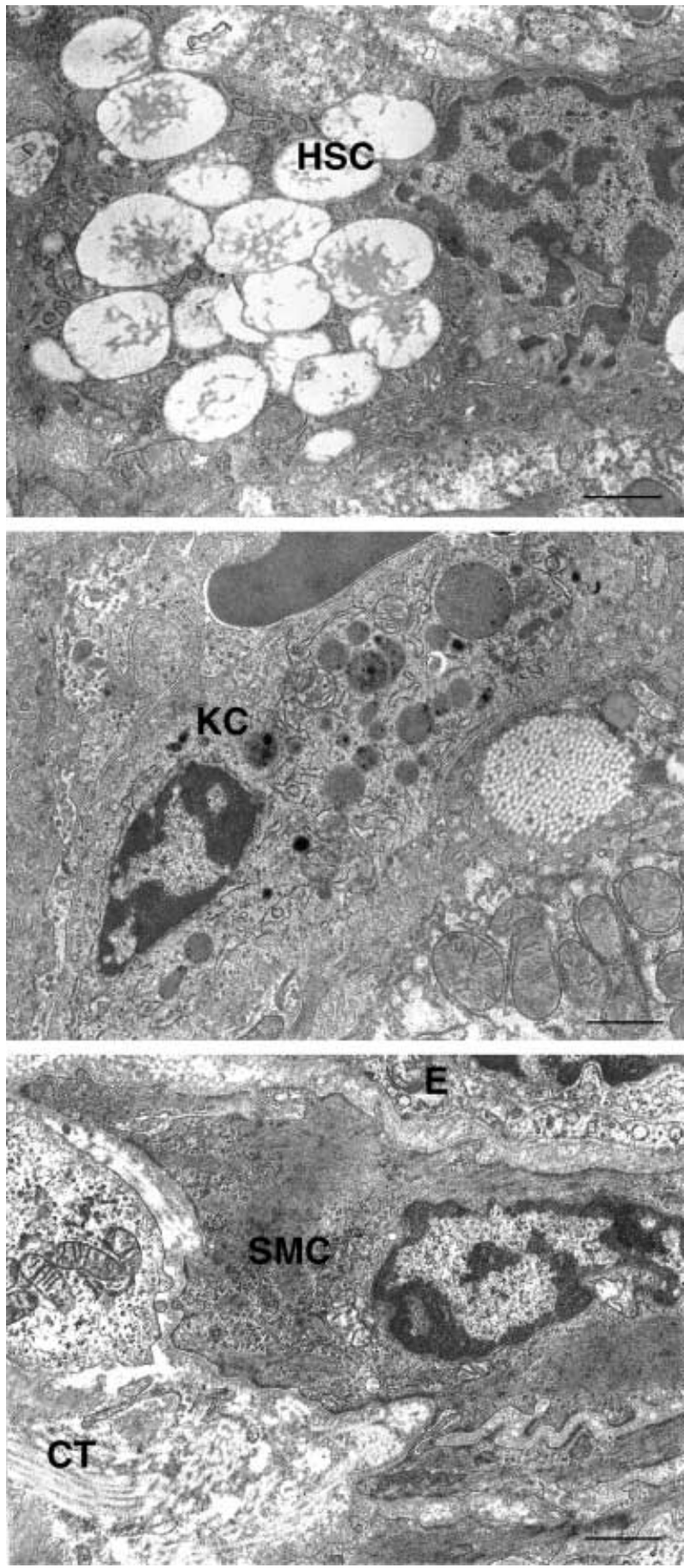

Figure 4 Control rat. (Upper panel) A hepatic stellate cell (HSC) with several lipid-containing cytoplasmic bodies can be seen in the Disse space. (Centre panel) A Kupffer cell (KC) containing several lysosomes and abutting into a sinusoid can be seen. (Lower panel) A microvessel in the portal space, likely a precapillary sphincter, shows non-fenestrated endothelium (E) close to a typical smooth muscle cell (SMC), showing abundant contractile filaments and a continuous basement membrane. CT: connective tissue. Electron micrographs, $\times 10700$, bars $=1 \mu \mathrm{m}$.
RLX-treated rats, actin-containing endothelial blebs were rare and small in size (Fig. 5B). Similar features were observed in the TDC-treated rats (data not shown).

The marked differences in the overall caliber of the sinusoids between the different experimental groups observed at visual examination were confirmed and quantitated by computer-assisted morphometry (Fig. 6). Compared with the controls, the mean surface area of the sinusoidal profiles was significantly greater in the RLXtreated rats and even more in the TDC-treated rats, whereas it was significantly smaller in the ethanol-treated rats. Of note, the value obtained in the rats which received L-NAME together with RLX was significantly lower than that of the rats given RLX alone.

\section{Discussion}

The results of this study showed that the endothelial lining of the sinusoids of the rat liver, besides typical endothelial cells, contains a peculiar type of endothelial cells that we have termed 'contractile endothelial cells' (CECs). These are characterized by a fairly developed contractile apparatus and cytoplasmic blebs protruding into the sinusoidal lumen. Therefore, in the liver microvessels, contractile cells potentially able to control hemodynamics are not limited to smooth muscle cells around the arterioles and precapillary sphincters or stellate cells located along the sinusoids (Zhang et al. 1995, Bauer et al. 2000), but also comprise CECs. Of note, CECs appear not to be restricted to the liver of rats, because such cells have also been observed in human liver sinusoids (authors' unpublished data). The present findings in the rats treated with substances known to increase or decrease sinusoidal blood flow, such as TDC and ethanol respectively (Hijioka et al. 1991, Pak \& Lee 1993), as well as with the vasorelaxant hormone RLX indicate that CECs can respond to bloodborne vasoactive agents reaching the liver, such as metabolites and hormones, by changing their contractile activity and consequently their overall shape. In particular, when vasodilation occurs CECs appear to flatten and to retract their cytoplasmic blebs, indicating that these cells may play an important role in regulating sinusoidal caliber and blood flow velocity. Until recently, the local and hormonal regulation of blood flow through the liver parenchyma had been mainly attributed to smooth muscle cells of arterioles and precapillary sphincters, whereas the existence of regulatory mechanisms within sinusoids had been poorly understood. An important contribution was made by McCuskey (1966) and McCuskey et al. (1983) who studied the rat liver in vivo by transillumination and first used the term 'sinusoidal sphincters' to indicate otherwise unspecified cellular devices held responsible for the fine regulation of sinusoidal blood flow. In recent years, microfluorographic investigations of liver microcirculation have provided evidence that hepatic stellate 

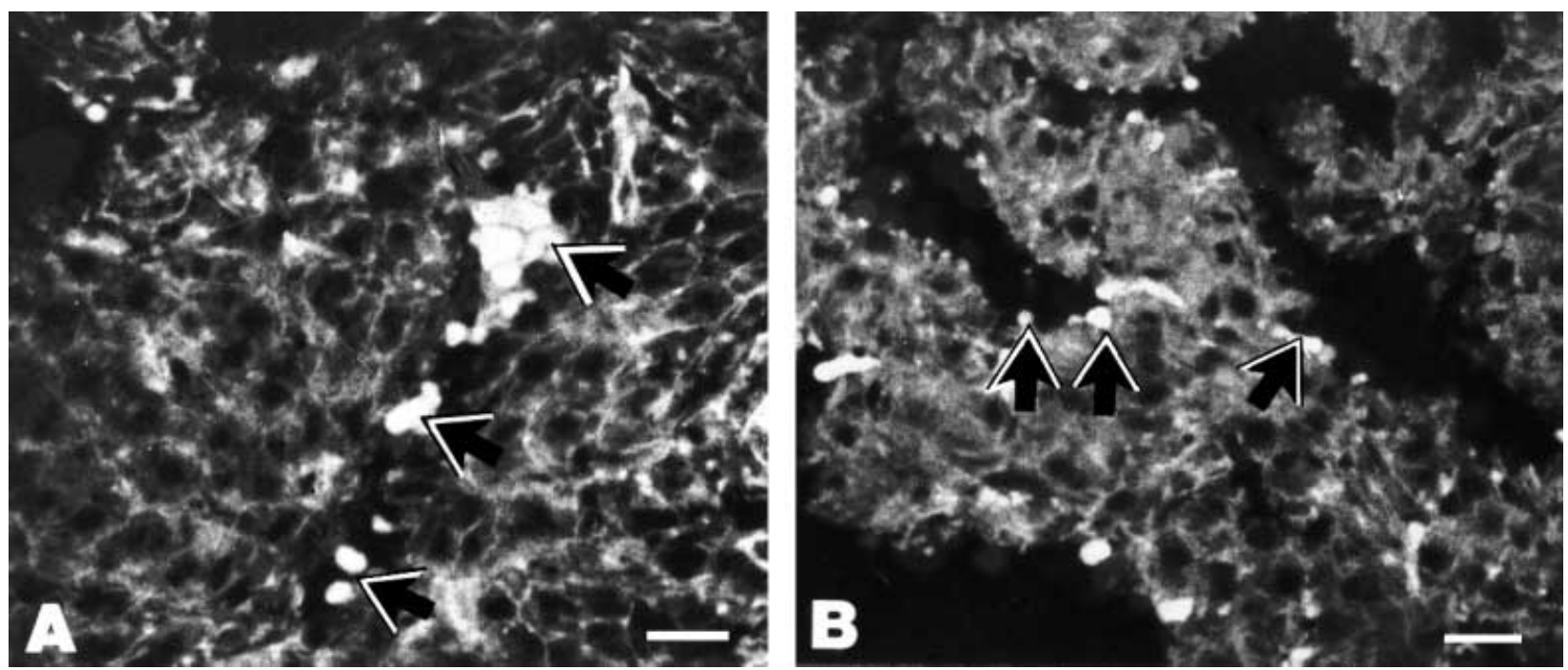

Figure 5 Cytochemical localization of actin by TRITC-labeled phalloidin. Representative liver sinusoids from (A) control rat: numerous endothelial blebs intensely labeled for actin are seen (arrows) and (B) RLX-treated rat: sparse, small-sized endothelial blebs labeled for actin are seen (arrows). Fluorescence light micrograph, $\times 780$, bars $=10 \mu \mathrm{m}$.

cells, which do have contractile properties (Ramadori 1990, Pinzani et al. 1992), can respond to vasoactive agents and thereby can participate in the regulation of sinusoidal blood flow (Suematsu et al. 1995, Zhang et al. 1995, Bauer et al. 2000). Our present study in the rat showed that

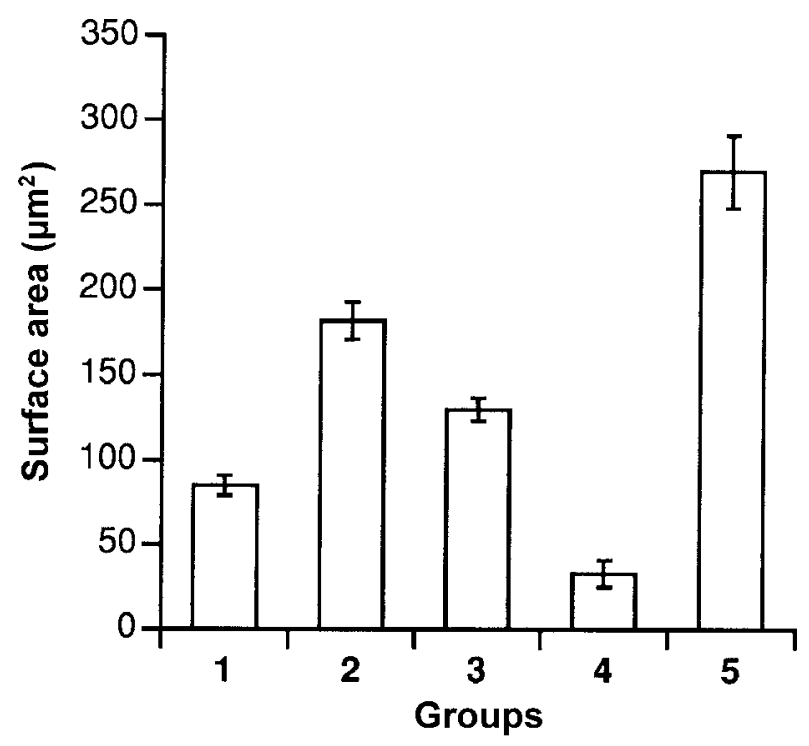

Figure 6 Histogram showing the mean \pm S.E.M. surface area of the sinusoidal profiles in the rats of the different experimental groups, evaluated by computer-assisted morphometry. 1, Control; 2, RLX; 3, L-NAME+RLX; 4, ethanol; 5, TDC. Significance of differences between the groups (one-way ANOVA test followed by StudentNewman-Keuls multiple comparison test): 1 vs $2, P<0 \cdot 001 ; 2$ vs 3 , $P<0.01 ; 1$ vs $3, P<0.001 ; 1$ vs $4, P<0 \cdot 001 ; 1$ vs $5, P<0.001$.
CECs also exist in liver sinusoids and provide support to the concept of endothelial sinusoidal sphincters acting as adjusting 'microscrews' of the local blood flow. In contrast to previous reports (McCuskey 1966, McCuskey et al. 1983), our findings show that CECs are not only located at the inlet and outlet of the sinusoids but extend for their overall length. One might ask why CECs of the sinusoids had escaped recognition in the numerous transmission and scanning electron microscopic studies of hepatic microcirculation. Indeed, these studies have been mainly performed in livers fixed by perfusion. It is likely that a supraphysiologic perfusion pressure may have artifactually dilated sinusoids and hence impeded recognition of these cells. However, it is worth noting that a previous scanning electron microscopic study of corrosion casts of hepatic microvessels has shown the occurrence of blind ends in branches of sinusoids (Kardon \& Kessel 1980). The authors hypothesized that these features may result from vessels with an occluded lumen but, in the absence of structural elements that could explain their observation, they were not able to validate this hypothesis. Our finding of bleb-like extensions of the CECs converging together to occlude the sinusoidal lumen may provide the morphological substrate for the above-reported unexplained observation.

The ultrastructural features of CECs clearly show that they are a cell type distinct from hepatic stellate cells, which are also deemed to be involved in the regulation of liver microcirculation. CECs are also substantially different from Kupffer cells, thus leading us to exclude the possibility that they may belong to the reticulo-endothelial system. 
Besides the recognition and characterization of a novel cell type in the liver sinusoids, the present study also provided the first experimental evidence that RLX causes dilation of liver sinusoids. In fact, in the RLX-treated rats, the mean surface area of these vessels was significantly higher than in the controls. Moreover, seen by both light and electron microscope, the endothelial lining of sinusoids was extremely flattened and the intralumenal blebs of CECs virtually disappeared. These findings, accompanied by filling of the sinusoidal lumen with blood cells, speak in favor of an RLX-induced increase in liver blood perfusion. This is in keeping with previous studies in which RLX has been repeatedly shown to dilate not only microvessels provided with a smooth muscle coat, such as arterioles, but also capillaries and postcapillary venules, characteristically lacking smooth muscle cells in their wall (Vasilenko et al. 1986, Bani et al. 1988, 1995, Bigazzi et al. 1988, Danielson et al. 1999). Based on these grounds, it is conceivable that CECs of the sinusoids are the principal target for RLX, especially considering that these cells are directly exposed to blood-borne vasoactive substances. Nevertheless, a relaxant action of RLX on other sinusoidal cells, such as common endothelial cells, hepatic stellate cells and Kupffer cells, as well as on smooth muscle cells of the arterial-type microvessels afferent to sinusoids can also be postulated. Indeed, RLX has been shown to be a potent relaxant for vascular smooth muscle cells (Bani Sacchi et al. 1995, Bani et al. 1998).

The current study also showed that L-NAME, a competitive NO-synthase inhibitor, significantly reduces the vasodilatory effect of RLX on liver sinusoids. This finding suggested that the response of the sinusoids to RLX involves a stimulation of the endogenous $\mathrm{NO}$ biosynthesis by the target cells, as also occurs for cardiac and renal microvessels (Bani Sacchi et al. 1995, Masini et al. 1997, Danielson et al. 1999).

It is well recognized that the pattern of liver microcirculation is a determinant of hepatocyte heterogeneity (Rappaport et al. 1954) and of the metabolic compartmentation within the different zones of the hepatic acinus (reviewed by Gumucio \& Miller 1982, Gumucio \& Chianale 1988). It is likely that the vasodilatory action of RLX may lead to an increase of blood flow and velocity and hence to changes in the gradients of solute and $\mathrm{pO}_{2}$ in the sinusoidal blood. These changes, in turn, can influence liver metabolism, as can be argued by our findings on the hepatocytes of the same RLX-treated rats as those studied here (Bani et al. 2001). In this context, the ability of RLX to increase hepatic circulation may be of some physiologic relevance during pregnancy when this hormone attains the highest plasma levels (Eddie et al. 1986). By this mechanism, RLX may participate in the metabolic changes of the liver occurring in pregnancy, which are directed to fulfil the fuel needs of the placenta and the conceptus (Paul 1972, Herrera \& Freinkel 1975, Baumann et al. 1981, Everson 1998, Freetly \& Ferrel 1998).

\section{Acknowledgements}

The authors gratefully acknowledge Dr O D Sherwood from the Department of Molecular and Integrative Physiology, University of Illinois at Urbana Champaign, Urbana, IL, USA, for having provided purified porcine relaxin as a gift. Many thanks are also due to Mr Patrizio Guasti, Department of Anatomy, Histology and Forensic Medicine, Florence, Italy, for skilful technical assistance. This work was supported by funds from the University of Florence.

\section{References}

Bani D 1997 Relaxin: a pleiotropic hormone. General Pharmacology 28 13-22.

Bani D, Failli P, Bello MG, Thiemermann C, Bani Sacchi T, Bigazzi M \& Masini E 1998 Relaxin activated the L-arginine-nitric oxide pathway in vascular smooth muscle cells in culture. Hypertension 31 1240-1247.

Bani D, Nistri S, Quattrone S, Bigazzi M \& Bani Sacchi T 2001 Relaxin causes changes of the liver. In vivo studies in rats. Hormone and Metabolic Research 33 175-180.

Bani G, Bani Sacchi T, Bigazzi M \& Bianchi S 1988 Effects of relaxin on the microvasculature of mouse mammary gland. Histology and Histopathology 3 337-343.

Bani G, Maurizi M, Bigazzi M \& Bani Sacchi T 1995 Effects of relaxin on the endometrial stroma. Studies in mice. Biology of Reproduction 53 253-262.

Bani Sacchi T, Bigazzi M, Bani D, Mannaioni PF \& Masini E 1995 Relaxin-induced increased coronary flow through stimulation of nitric oxide production. British Journal of Pharmacology 116 1589-1594.

Bauer M, Paquette NC, Zhang JX, Bauer I, Pannen BH, Kleeberger SR \& Clemens MG 1995 Chronic ethanol consumption increases hepatic sinusoidal contractile response to endothelin-1 in the rat. Hepatology 22 1565-1576.

Bauer M, Bauer I, Sonin NV, Kresge N, Baveja R, Yokohama Y, Harding D, Zhang JX \& Clemens MG 2000 Functional significance of endothelin $\mathrm{B}$ receptors in mediating sinusoidal and extrasinusoidal effects of endothelins in the intact rat liver. Hepatology 31 937-947.

Baumann G, Puavilai G, Freinkel N, Domont LA, Metzger BE \& Levene HB 1981 Hepatic insulin and glucagon receptors in pregnancy: their role in the enhanced catabolism during fasting. Endocrinology 108 1979-1986.

Bigazzi M, Del Mese A, Petrucci F, Casali R \& Novelli GP 1986 The local administration of relaxin induces changes in the microcirculation in the rat mesocaecum. Acta Endocrinologica 112 296-299.

Bigazzi M, Bani G, Bani Sacchi T, Petrucci F \& Bianchi S 1988 Relaxin: a mammotrophic hormone promoting growth and differentiation of the pigeon crop sac mucosa. Acta Endocrinologica 117 181-188.

Campra JL \& Reynolds TB 1988 The hepatic circulations. In The Liver: Biology and Pathology, edn 2, pp 911-930. Eds IM Arias, WB Jakoby, H Popper, D Schachter \& DA Shafritz. New York: Raven Press.

Danielson LA, Sherwood OD \& Conrad KP 1999 Relaxin is a potent renal vasodilator in conscious rats. Journal of Clinical Investigation 103 $525-533$.

Eddie LW, Lester A, Bennett G, Bell RJ, Geier M, Johnston PD \& Niall HD 1986 Radioimmunoassay of relaxin in pregnancy with an analogue of human relaxin. Lancet $\mathbf{i}$ (8494) 1344-1346. 
Everson GT 1998 Liver problems in pregnancy: distinguishing normal from abnormal hepatic changes. Medscape Womens Health 3 www.medscape.com/medscape/womenshealth

Freetly HC \& Ferrel CL 1998 Net flux of glucose, lactate, volatile fatty acid, and nitrogen metabolites across the portal-drained viscera and liver of pregnant cives. Journal of Animal Science 76 3133-3145.

Gumucio JJ \& Miller DL 1982 Liver cell heterogeneity. In The Liver: Biology and Pathology, pp 647-661. Eds IM Arias, H Popper, D Schachter \& DA Shafritz. New York: Raven Press.

Gumucio JJ \& Chianale J 1988 Liver cell heterogeneity and liver function. In The Liver: Biology and Pathology, edn 2, pp 931-947. Eds IM Arias, WB Jakoby, H Popper, D Schachter \& DA Shafritz. New York: Raven Press.

Herrera E \& Freinkel N 1975 Metabolites in the liver brain and placenta of fed or fasted mothers and fetal rats. Hormone and Metabolic Research 7 247-249.

Hijioka T, Sato N, Matsumura T, Yoshihara H, Takei Y, Fukui H, Oshita M, Kawano S \& Kamada T 1991 Ethanol-induced disturbance of hepatic microcirculation and hepatic hypoxia. Biochemical Pharmacology 41 1551-1557.

Kardon RH \& Kessel RG 1980 Three-dimensional organization of the hepatic microcirculation in the rodent as observed by scanning electron microscopy of corrosion casts. Gastroenterology 79 72-81.

McCuskey RS 1966 A dynamic and static study of hepatic arterioles and hepatic sphincters. American Journal of Anatomy 119 455-478.

McCuskey RS, Urbaschek R, McCuskey PA \& Urbaschek B 1983 In vivo microscopic observations of the responses of Kupffer cells and the hepatic microcirculation to Mycobacterium bovis BCG alone and in combination with endotoxin. Infection and Immunity $\mathbf{4 2}$ 362-367.

Masini E, Bani D, Bello MG, Bigazzi M, Mannaioni PF \& Bani Sacchi T 1997 Relaxin counteracts myocardial damage induced by ischemia-reperfusion in isolated guinea pig hearts: evidence for an involvement of nitric oxide. Endocrinology 138 4713-4720.

Mittal MK, Gupta TK, Lee FY, Sieber CC \& Groszmann RJ 1994 Nitric oxide modulates hepatic vascular tone in normal rat liver. American Journal of Physiology 267 G416-G422.

Norrby K, Bani D, Bigazzi M \& Bani Sacchi T 1996 Relaxin, a potent microcirculatory effector, is not angiogenic. International Journal of Microcirculation 16 227-231.

Orrego H, Mena I, Baraona E \& Palma R 1965 Modifications in hepatic blood flow and portal pressure produced by different diets. American Journal of Digestive Diseases 10 239-248.
Pak JM \& Lee SS 1993 Vasoactive effects of bile salts in cirrhotic rats: in vivo and in vitro studies. Hepatology 18 1175-1181.

Paul PK 1972 Dynamics of hepatic glycogen: oestrogen and pregnancy. Acta Endocrinologica 71 385-392.

Pinzani M, Failli P, Ruocco C, Casini A, Milani S, Baldi E, Giotti A \& Gentilini P 1992 Fat-storing cells as liver-specific pericytes. Spatial dynamics of agonist-stimulated intracellular calcium transients. Journal of Clinical Investigation 90 642-646.

Ramadori G 1990 The stellate cell (Ito cell, fat-storing cell, lipocyte, perisinusoidal cell) of the liver: new insights into pathophysiology of an intriguing cell. Virchows Archiv B Cell Pathology 59 147-158.

Rappaport AM 1973 The microcirculatory hepatic unit. Microvascular Research 6 212-228.

Rappaport AM 1980 Hepatic blood flow: morphologic aspects and physiologic regulation. International Review of Physiology 21 1-63.

Rappaport AM \& Schneiderman JH 1976 The function of the hepatic artery. Review of Physiology, Biochemistry and Pharmacology 76 129-175.

Rappaport AM, Borowy ZJ, Lougheed WM \& Lotto WN 1954 Subdivision of hexagonal liver lobules into a structural and functional unit. Role in hepatic physiology and pathology. Anatomical Record 119 11-13.

Sherwood OD \& O'Byrne EM 1974 Purification and characterization of porcine relaxin. Archives of Biochemistry and Biophysics $\mathbf{6 0}$ 185-196.

Shoemaker WC, Yanof HM, Turk LN \& Wilson TH 1963 Glucose and fructose absorption in the unanesthetized dog. Gastroenterology 44 654-663.

Suematsu M, Goda N, Sano T, Kashiwagi S, Egawa T, Shinoda Y \& Ishimura Y 1995 Carbon monoxide: an endogenous modulator of sinusoidal tone in the perfused rat liver. Journal of Clinical Investigation 96 2431-2437.

Vasilenko P, Mead JP \& Weidmann JE 1986 Uterine growthpromoting effects of relaxin: a morphometric and histological analysis. Biology of Reproduction 35 987-996.

Zhang JX, Bauer M \& Clemens MG 1995 Vessel- and target cellspecific actions of endothelin-1 and endothelin-3 in rat liver. American Journal of Physiology (Gastrointestinal Liver Physiology 32) 269 G269-G277.

Received 17 May 2001

Accepted 16 August 2001 